\title{
Effects of drugs on postprandial lipoprotein metabolism
}

\author{
BY C. J. PACKARD
}

Department of Pathological Biochemistry, Institute of Biochemistry, Glasgow G4 OSF

Inefficient chylomicron clearance following a fatty meal has been linked with increased risk of CHD. Zilversmit (1979) was the first to postulate that atherosclerosis may be promoted by postprandial lipoproteins, particularly cholesteryl ester-enriched chylomicron remnants. A number of studies have shown subsequently that when patients with CHD are given a standard fat-load test meal they develop an exaggerated alimentary lipaemia (Simpson et al. 1990; Groot et al. 1991). In an early report from our laboratory, Simpson et al. (1990) demonstrated increased plasma chylomicron levels following a fat-load test in subjects with angiographically-proven coronary artery disease, even when those with elevated fasting lipid levels were excluded so that controls and cases had similar fasting plasma triacylglycerol. This is a key observation since fasting triacylglycerol level is a major determinant of alimentary lipaemia and is often not controlled for in comparative studies. These data indicate that CHD may be associated with a defect in chylomicron metabolism that does not dramatically influence VLDL turnover.

There are several key steps in chylomicron production, lipolysis and particle clearance where drugs may conceivably operate. First, chylomicrons are elaborated in the gut in response to the absorption of dietary fat, principally triacylglycerol and cholesterol. These lipids must first be assimilated by enterocytes and this requires the breakdown of triacylglycerol to fatty acids and partial acylglycerols by the action of pancreatic lipase ( $E C$ 3.1.1.3) and the release of cholesterol from its ester by an esterase. Blocking the activity of these enzymes would be predicted to have a potent effect on plasma and whole-body lipid levels. However, attempts to use compounds to block absorption have so far yielded disappointing results. Chylomicrons are large triacylglycerol-rich particles that, when released from enterocytes, pass into the systemic circulation and are there acted on by lipoprotein lipase (EC 3.1.1.34; LPL) which hydrolyses the core triacylglycerol and, hence, delivers fatty acids to tissues such as skeletal muscle and adipose beds. LPL is a highly regulated enzyme under hormonal control, particularly by insulin (Taskinen \& Kuusi, 1987). Drugs are available to perturb its activity as described later and they have a profound effect on chylomicron clearance, emphasizing the key regulatory importance of this lipolytic step. Following removal of most of the triacylglycerol from the chylomicron particle a remnant is formed which is cleared rapidly by receptor-mediated processes in the liver. Genetic experiments in mice have provided strong evidence that both the LDL receptor and the LDL-receptor-related protein are involved in remnant removal (Ishibashi et al. 1994). These receptors recognize apolipoprotein (apo) $E$ on the surface of the lipoprotein and thereby bind the particle and facilitate its endocytosis. This apo E-receptor interaction is inhibited by a second apolipoprotein, apo C-III, through an as yet unknown mechanism so that chylomicron remnant clearance can be linked to the apo E: apo C-III on the lipoprotein (Aalto-Setälä et al. 1992). This ratio is amenable to change through the action of drugs that affect apo C-III production and at least in animal models this change has been shown to have consequences for the rate of remnant clearance.

It is difficult to perform classical steady-state kinetic experiments to investigate alimentary lipaemia, since the plasma level of chylomicrons changes continuously and the rate of clearance of particles is approximately the same as their rate of release from the gut. 
Chylomicron kinetics have been studied either by quantifying the area under the plasma triacylglycerol increment following a standard fat-load test or by including retinol in the meal and following its appearance and disappearance from the circulation. This vitamin in its esterified form is secreted from the gut in chylomicrons, is believed to undergo little exchange with other lipoproteins, is cleared by the liver and does not become incorporated into other hepatically-generated lipoproteins. Thus, it acts as a marker for the particle's fate in the bloodstream. A further method for analysing the metabolism of gut-derived particles is to follow the kinetics of apo B-48, the isoform of apo B produced specifically by the intestine in human subjects. The following discussion describes the action of each major class of lipid-lowering drug in plasma chylomicron metabolism, and the further consequences that changes in this pathway may have on other lipoproteins.

\section{FIBRATES AND POSTPRANDIAL LIPOPROTEIN METABOLISM}

The fibrate group of drugs are related chemically to the parent compound, clofibrate. They have been widely used for many years as effective agents for reducing plasma triacylglycerol and cholesterol concentrations. They also exhibit the property of elevating HDL by $10-30 \%$. Their major effect is to lower plasma triacylglycerol and VLDL levels by $50-70 \%$ through a combination of inhibiting VLDL production in liver and accelerating its clearance. Given this mechanism of action it is not surprising that fibrates have a profound effect on postprandial lipaemia. Weintraub et al. (1987) showed that gemfibrozil reduced the incremental area under the plasma triacylglycerol, chylomicron triacylglycerol and plasma retinyl palmitate (RP) curves following a fatty test meal in both hypertriacylglycerolaemic and normotriacylglycerolaemic, hypercholesterolaemic subjects. The area under the RP curve in control normals and the hypercholesterolaemic subjects was inversely related to post-heparin plasma LPL activity and HDL-cholesterol concentration. However, these correlations were much weaker in hypertriacylglycerolaemics, indicating other factors may play a more dominant regulatory role. In the latter group of subjects, fasting plasma triacylglycerol was the strongest predictor of chylomicron RP area under curve. Plasma triacylglycerol in the fasting state was also the main determinant of the response to a fat-load test in a study of normal and hyperlipidaemic subjects with and without CHD, although as noted previously having coronary artery disease was also associated with an exaggerated alimentary lipaemia independent of fasting triacylglycerol level (Simpson et al. 1990).

In our study (Simpson et al. 1990) fenofibrate reduced the area under the lipoprotein (density $<1.006 \mathrm{~g} / \mathrm{ml}$ ) curve by about $50 \%$ but did not markedly affect the RP response. We interpreted this action of the drug as a promotion of lipolysis and, hence, triacylglycerol removal from chylomicron particles but particle clearance was unaffected. This finding was contrary to the earlier report of Weintraub et al. (1987). Plasma postheparin LPL activity increased $20 \%$ in our study but there was no convincing relationship between the change in LPL and the reduction in triacylglycerol area under the curve.

Subjects with underlying primary causes of hypertriacylglycerolaemia, i.e. due to raised VLDL levels, also exhibit defective chylomicron metabolism. Non-insulindependent diabetics show an abnormal response to a standard fat load that can be corrected by fibrate treatment. In the study of Syvänne et al. (1993) gemfibrozil caused a $38 \%$ decrease in the area under the chylomicron triacylglycerol curve and a similar decrease in the increment observed in Svedberg flotation unit $\left(S_{f}\right) 60-400$ lipoproteins after the test meal. VLDL levels in the $S_{f} 20-60$ density interval were unaffected by therapy. In contrast with earlier studies these authors reported no association between postprandial 
lipaemia and HDL-cholesterol levels or post-heparin plasma LPL activity. However, they did observe significant correlations between the cholesterol : triacylglycerol value in HDL and the RP area under the curve, i.e. high levels of chylomicrons and their remnants were associated with triacylglycerol enrichment in HDL. In contrast with other studies, in diabetics baseline (fasting) VLDL-triacylglycerol was not a predictor of the incremental area under the plasma triacylglycerol curve following the fat meal, and on the basis of this observation Syvänne et al. (1993) speculated that the relationships between VLDL and chylomicron metabolism may differ in diabetic $v$. non-diabetic subjects.

\section{Chylomicron and HDL metabolic interrelationships}

There is a well-known inverse relationship between plasma triacylglycerol and HDLcholesterol in the population that is believed to arise because of a link between the metabolism of HDL and triacylglycerol-rich lipoproteins. Patsch et al. (1987) showed that this link extended to chylomicrons by following the changes in HDL lipid composition, size and apoprotein content during alimentary lipaemia. As the concentration of chylomicrons rises following a fatty meal, there is a transfer of triacylglycerol from these lipoproteins into HDL while cholesterol ester moves in the opposite direction. This neutrallipid exchange is mediated by cholesteryl ester transfer protein (CETP). Triacylglycerolenriched HDL is subject to the action of hepatic lipase (HL), with the result that both core (triacylglycerol) and surface (phospholipid) material is removed from the particle and it shrinks in size. A consequence of this change in HDL structure is a conformational change in apo A-I on the surface of HDL so that it is more likely to be lost from the particle and undergo catabolism (Brinton et al. 1994). This sequence of events explains the relationships between HL:LPL, apo A-I fractional catabolic rate and the HDLcholesterol : apo A (the latter is a function of HDL size; Brinton et al. 1994). We (Simpson et al. 1990) followed lipid changes in HDL following a fat-load test on the subjects before and during fenofibrate treatment. In those receiving the drug, HDLcholesterol levels were higher in the fasting state and less cholesteryl ester was lost from this lipoprotein during the period of alimentary lipaemia, presumably because fewer triacylglycerol-rich lipoproteins were present to promote CETP-mediated lipid exchange. It is likely that these changes in postprandial metabolism underlie the rise in HDL seen on fibrate therapy.

\section{Mechanism of action of fibrates}

Fibrates have a wide range of actions on lipoprotein metabolism and until recently it was difficult to conceive of a simple mechanism whereby they achieved their effect. The discovery that these drugs can activate (directly or indirectly) peroxisome proliferatoractivated receptors (PPAR), members of a family of steroid hormone nuclear receptors, has provided the much sought after molecular basis for the effects of these drugs on lipids (Schoojans et al. 1996). A number of genes whose products are involved in lipid homeostasis are regulated by PPAR. These include acyl-CoA carboxylase, LPL, apo A-I and apo C-III. Fibrates acting via PPAR suppress the transcription of apo C-III and promote LPL synthesis. The combined effect of these changes is predicted to be exactly what was noted previously, i.e. promotion of chylomicron lipolysis and enhanced remnant particle uptake in the liver (Fig. 1). 


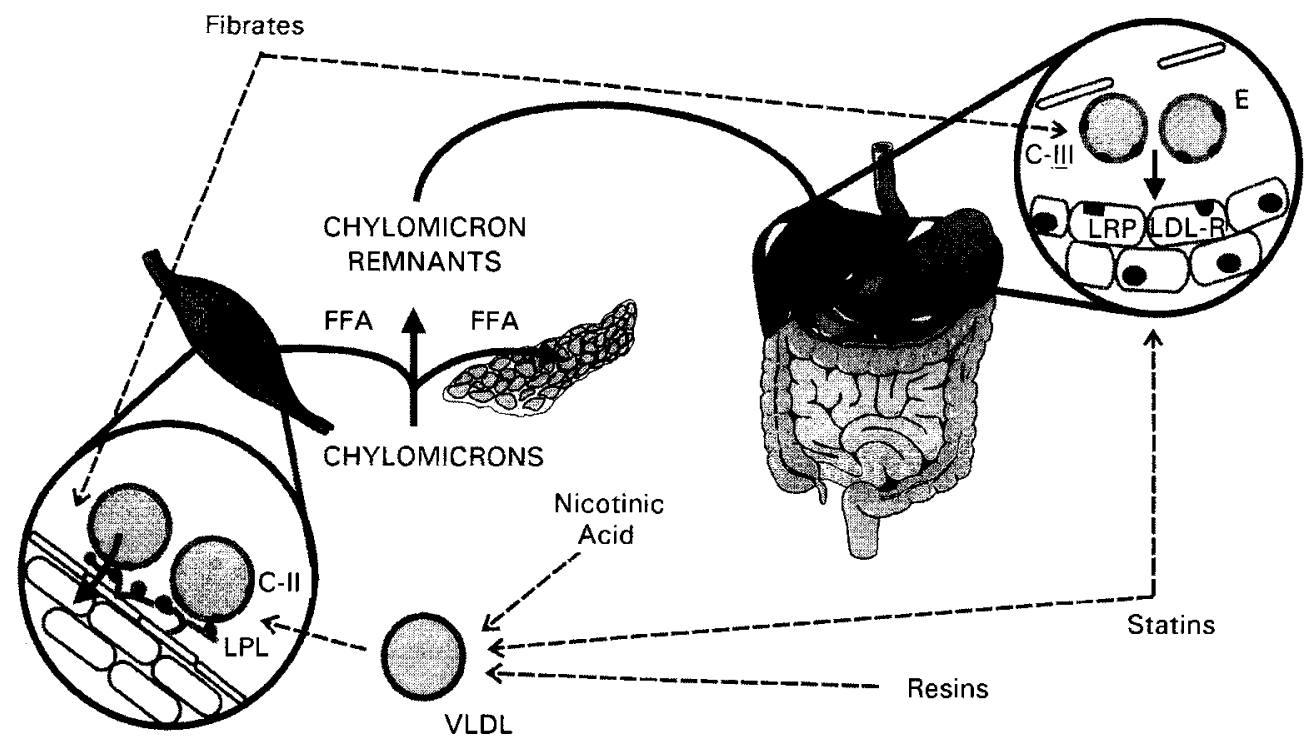

Fig. 1. Chylomicrons elaborated in the gut are cleared by a two-step mechanism. Lipoprotein lipase (EC 3.1.1.34; LPL) bound to cell-surface glycosaminoglycans is situated on the capillary endothelium of skeletal muscle and adipose tissue. Chylomicron-core triacylglycerol hydrolysis occurs rapidly when LPL interacts with its co-factor apolipoprotein (apo) $\mathrm{C}$-II on the surface of the particle. Apo C-III (C-III) inhibits this action. When lipolysis nears completion a remnant is formed which is taken up rapidly by the liver via receptor-mediated endocytosis. This is believed to involve both the LDL receptor (LDL-R) and the LDL-R-related protein (LRP). Fibrates promote synthesis of LPL and inhibit the transcription of C-III, actions which stimulate lipolysis and also enhance the rate of remnant clearance by the liver. Statins may promote chylomicron clearance by inducing expression of LDL receptors but, like resins and nicotinic acid, more probably affect alimentary lipaemia by changing the level of VLDL in plasma. VLDL competes with chylomicrons for lipolytic sites and when present in excess will reduce the efficiency of chylomicron triacylglycerol hydrolysis by LPL. FFA, free fatty acids.

\section{STATINS AND POSTPRANDIAL LIPOPROTEINS}

The main indication for statin prescription is primary hypercholesterolaemia and this class of drugs has only a modest effect on plasma triacylglycerol and VLDL levels. Typical reductions in triacylglycerol on higher doses of simvastatin or pravastatin are 10-20\%. However, it has been consistently reported that in moderate combined hyperlipidaemia more substantial changes in plasma triacylglycerol occur when statins are given, of the order of $20-40 \%$ (Fuessner, 1994). The reason for this is not clear since the drugs have a known, presumably single, mode of action which is inhibition of hydroxymethylglutarylCoA reductase ( $E C$ 1.1.1.88), the rate-limiting enzyme in cholesterol synthesis. Relatively few investigations have been performed on the effects of statins on postprandial lipid metabolism. Weintraub et al. (1989) were the first to show that alimentary lipaemia following a fat-load test was diminished by about $50 \%$ (area under chylomicron RP curve) on high-dose lovastatin treatment at $80 \mathrm{mg} / \mathrm{d}$. They made the important observation that, unlike fibrate, statins had no effect on chylomicron metabolism in normotriacylglycerolaemic subjects (plasma triacylglycerol $<1.8 \mathrm{mmol} / \mathrm{l}$ ). Lipase activities were measured and neither LPL nor hepatic lipase were affected by treatment. The major determinant of the area below the chylomicron RP curve both before and on lovastatin was the fasting plasma triacylglycerol level. Simo et al. (1993) conducted a direct comparison of the 
effects of gemfibrozil $(600 \mathrm{mg} / \mathrm{d})$ and lovastatin $(40 \mathrm{mg} / \mathrm{d})$ in men with moderate hypertriacylglycerolaemia and low HDL levels. As predicted, the fibrate was superior at lowering plasma triacylglycerol $(55 \% v .22 \%$ on statin) but the statin produced a greater fall in plasma cholesterol. Gemfibrozil caused a $50 \%$ reduction in alimentary lipaemia in these subjects, while lovastatin treatment led to a more modest $20 \%$ reduction which was not significant, although the trend was clear. In comparing these two studies (Weintraub et al. 1989; Simo et al. 1993), the differing effect of lovastatin on triacylglycerol levels in the two groups is noteworthy (although the dose of drug was not the same). Castro Cabezas $e t$ al. (1993) demonstrated once again the potential value of statin treatment (simvastatin $10-40 \mathrm{mg} / \mathrm{d}$ ) on chylomicron clearance, this time in familial combined hyperlipidaemic patients in whom the drug reduced the area under the chylomicron RP curve by more than $50 \%$. These authors related the change in chylomicron clearance to the fall in both apo $\mathrm{E}$ and apo C-III in plasma.

It is unlikely that statins affect chylomicron clearance directly by influencing fat absorption or the activity of LPL. Furthermore, they appear to have a significant effect only when the lipolytic capacity of the subject is under stress, i.e. when VLDL levels are elevated. Two possible mechanisms for their action may be considered. First, by promoting LDL receptor activity they enhance remnant clearance by the liver, since the receptor has been shown to participate in this final catabolic step. Second, by lowering fasting VLDL levels the drugs reduce competition for lipase sites and permit more efficient binding of the chylomicrons to the enzyme in adipose tissue and skeletal muscle (Fig. 1). At present it is not clear which of these two is most important and it is probable that both contribute to the effects observed. The mechanism by which statins lower VLDL is also unclear, with both increased particle clearance and decreased production in the liver being postulated as candidate actions.

\section{EFFECTS OF OTHER LIPID-LOWERING AGENTS}

Nicotinic acid derivatives, as agents that lower plasma triacylglycerol, would be expected to reduce lipaemia following a fat-load test, and this is indeed the case. Pazzucconi et al. (1993) showed that short-term treatment with acipimox lowered the area under the curve for triacylglycerol in chylomicrons plus VLDL by $16 \%$. We, (Simpson, Packard and Shepherd, unpublished results), confirmed this finding using standard nicotinic acid for 6 weeks. On treating normal subjects with the drug, fasting plasma triacylglycerol fell $40 \%$ and the area under the plasma triacylglycerol curve following the standard fat-load test fell by $44 \%(P<0.05)$. Interestingly, post-heparin plasma LPL fell $9.5 \%(P<0.02)$ while hepatic lipase activity was unaltered. Pazzucconi et al. (1993) also explored the acute changes in size of HDL and LDL during alimentary lipaemia. The same perturbation in HDL was observed whether or not subjects received acipimox, in that the content of $\mathrm{HDL}_{2 b}$ (the largest fraction) fell during alimentary lipaemia while that of $\mathrm{HDL}_{3 b}$ (a dense $\mathrm{HDL}_{3}$ subspecies) rose. This is concordant with the findings of Patsch et al. (1987). In contrast, LDL size, as measured by particle diameter on gradient gels, was not altered by the influx of chylomicrons.

Cholestyramine is a bile acid-sequestering resin that, like statins, lowers LDL by promoting LDL-receptor-mediated catabolism in liver. However, unlike statins, resins tend to increase plasma triacylglycerol and VLDL levels, especially the plasma concentration of the largest, most triacylglycerol-rich VLDL. This is thought to be due to a stimulation of hepatic triacylglycerol synthesis and enhanced VLDL release from the liver as a result of release of inhibition of phosphatidate phosphatase (EC 3.1.3.4) by bile acid (Packard \& 
Shepherd, 1982). In the study of Weintraub et al. (1989), in hypercholesterolaemic subjects, cholestyramine increased the area under the plasma RP curve by $86 \%$ after a fatload test. This rather unusual effect is probably the result of increased competition for lipolytic sites by VLDL during resin therapy (Fig. 1), since the drug does not alter LPL activity (Weintraub et al. 1989) or other features of chylomicron metabolism. There are few data available on other lipid-lowering compounds. Probucol which has a unique mode of action in lowering plasma cholesterol, had been studied in a rat model. The drug is known not to affect plasma triacylglycerol levels in human subjects (Atmeh et al. 1983) and in normal rats does not perturb chylomicron clearance from plasma. However, if the rats are made diabetic with streptozotocin, then fasting plasma triacylglycerol levels are elevated and chylomicron clearance delayed (Mamo et al. 1993). Treatment with probucol both reduced fasting triacylglycerol levels and stimulated clearance of chylomicrons. The mechanism of this effect is unclear but may be related to the finding that probucol alters the apo E : apo C-III value in triacylglycerol-rich lipoproteins (Mamo et al. 1993).

\section{CONCLUSIONS}

Drugs perturb the metabolism of chylomicrons largely, it appears, as a function of the extent to which they alter fasting VLDL levels. The concentration of plasma triacylglycerol in the fasting state is, in most instances, the strongest predictor of the efficiency of chylomicron clearance. However, less than $50 \%$ of the inter-individual variance in the area under the chylomicron triacylglycerol or RP curve is explained by differences in plasma triacylglycerol, thus other major factors which control the residence time of chylomicrons in the bloodstream must exist, and remain to be elucidated. Possibilities include the apo CIII content of particles which regulates both lipolysis and particle uptake and the activity of various receptors in the body. The statin effects in hypertriacylglycerolaemics indicate that receptor number as well as the properties of the ligand lipoprotein may be critical in determining the clearance of these potentially-atherogenic lipoproteins from the bloodstream.

The help of Mrs Nancy Thomson in the preparation of this manuscript is gratefully acknowledged.

\section{REFERENCES}

Aalto-Setälä, K., Fisher, E. A., Chen, X., Chajek-Shaul, T., Hayek, T., Zechner, R., Walsh, A., Ramakrishnan, R., Ginsberg, H. N. \& Breslow, J. L. (1992). Mechanism of hypertriglyceridemia in human apolipoprotein (apo) CIII transgenic mice. Diminished very low density lipoprotein fractional catabolic rate associated with increased apo-CIII and reduced apo E on the particles. Journal of Clinical Investigation 90, 1889-1900.

Atmeh, R. F., Stewart, J. M., Boag, D., Packard, C. J., Lorimer, A. R. \& Shepherd, J. (1983). The hypolipidemic action of procubol: A study of its effects on high and low density lipoproteins. Journal of Lipid Research 24, 588-595.

Brinton, E. A., Eisenberg, S. \& Breslow, J. L. (1994). Human HDL cholesterol levels are determined by apo AI fractional catabolic rate, which correlates inversely with estimates of HDL particle size. Arteriosclerosis \& Thrombosis 14, 707-720.

Castro Cabezas, M., de Bruin, T. W. A., Jansen, H., Kock, L. A. W., Kortlandt, W. \& Erkelens, D. W. (1993). Impaired chylomicron remnant clearance in familial combined hyperlipidemia. Arteriosclerosis \& Thrombosis 13, 804-814.

Fuessner, G. (1994). HMG CoA reductase inhibitors. Current Opinion in Lipidology 5, 59-68.

Groot, P. H. E., van Stiphout, W. A. H. J., Krauss, X. H., Jansen, H., van Tol, A., van Ramshorst, E., Chin-On, S., Hofman, A., Cresswell, S. R. \& Havekes, L. (1991). Postprandial lipoprotein metabolism in normolipidemic men with and without coronary artery disease. Arteriosclerosis \& Thrombosis 11, 653-662. 
Ishibashi, S., Herz, J., Maeda, N., Goldstein, J. L. \& Brown, M. S. (1994). The two receptor model of lipoprotein clearance: Tests of the hypothesis in 'knockout' mice lacking the low density lipoprotein receptor, apolipoprotein E or both proteins. Proceedings of the National Academy of Sciences USA 91, 4431-4435.

Mamo, J. C. L., Elsegood, C. L., Umeda, Y., Hirano, T. \& Redgrave, T. G. (1993). Effect of probucol on plasma clearance and organ uptake of chylomicrons and VLDLs in normal and diabetic rats. Arteriosclerosis \& Thrombosis 13, 231-239.

Packard, C. J. \& Shepherd, J. (1982). The hepatobiliary axis and lipoprotein metabolism effects of bile acid sequestrants and ileal bypass surgery. Journal of Lipid Research 23, 1081-1098.

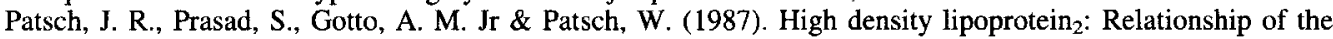
plasma levels of this lipoprotein species to its composition, to the magnitude of postprandial lipemia and to the activities of lipoprotein lipase and hepatic lipase. Journal of Clinical Investigation 80, 341-347.

Pazzucconi, F., Franceschini, G., Gianfranceschi, G., Brambilla, E. \& Sirtori, C. R. (1993). Lipoprotein structure in male subjects during in vivo lipolysis: effect of an anti-lipolytic treatment with acipimox. Journal of Lipid Research 34, 1465-1472.

Schoojans, K., Staels, B. \& Auwerx, J. (1996). Role of the peroxisome proliferator-activated receptor (PPAR) in mediating the effects of fibrates and fatty acids on gene expression. Joumal of Lipid Research 37, $907-925$.

Simo, I. E., Yakichuk, J. A \& Ooi, T. C. (1993). Effect of gemfibrozil and lovastatin on postprandial lipoprotein clearance in the hypoalphalipoproteinemia and hypertriglyceridemia syndrome. Atherosclerosis 100, 55-64.

Simpson, H. S., Williamson, C. M., Olivecrona, T., Pringle, S., Maclean, J., Lorimer, A. R., Bonnefous, F., Bogaievsky, Y., Packard, C. J. \& Shepherd, J. (1990). Postprandial lipemia, fenofibrate and coronary artery disease. Atherosclerosis 85, 193-202.

Syvänne, M., Vuorinen-Markola, H., Hilden, H. \& Taskinen, M.-R. (1993). Gemfibrozil reduces postprandial lipaemia in non-insulin dependent diabetes mellitus. Arteriosclerosis \& Thrombosis 13, 286-295.

Taskinen, M.-R. \& Kuusi, T. (1987). Enzymes involved in triglyceride hydrolysis. Baillières Clinical Endocrinology and Metabolism 1, 639-666.

Weintraub, M. S., Eisenberg, S. \& Breslow, J. L. (1987). Different patterns of postprandial lipoprotein metabolism in normal, type IIa, type III and type IV hyperlipoproteinemic individuals. Effects of treatment with cholestyramine and gemfibrozil. Journal of Clinical Investigation 79, 1110-1119.

Weintraub, M. S., Eisenberg, S. \& Breslow, J. L. (1989). Lovastatin reduces postprandial lipoprotein levels in hypercholesterolaemic patients with mild hypertriglyceridaemia. European Journal of Clinical Investigation $19,480-485$

Zilversmit, D. B. (1979). Atherogenesis: A postprandial phenomenon. Circulation 60, 473-485. 